\title{
THE EDUCATIONAL TRAJECTORY OF HIGH SCHOOL STUDENTS TO ENGINEERING PROGRAMS AT A COMPREHENSIVE CANADIAN UNIVERSITY
}

\author{
Scott Davies ${ }^{1}$, Qin Liu' ${ }^{2}$, and Greg Evans ${ }^{3}$ \\ ${ }^{1}$ Ontario Institute for Studies in Education, University of Toronto \\ ${ }^{2,3}$ Institute for Studies in Transdisciplinary Engineering Education and Practice, Faculty of Applied Science \& \\ Engineering, University of Toronto \\ Corresponding Author E-mail Addresses: scott.davies@,utoronto.ca; qinql.liu@,utoronto.ca; greg.evans@utoronto.ca
}

\begin{abstract}
This paper reports an analysis of an integrated data set that longitudinally tracked over 14,000 students from Canada's largest school board (the Toronto District School Board [TDSB]) into Canada's largest university (the University of Toronto [UofT]). Our analysis showed that when controlling for high school academic records and other student demographics, immigrant students from TDSB were more likely to pursue engineering than other fields of study; math ability was a strong predictor for the TDSB-UofT Engineering pathway; and while engineering students had better academic outcomes than other students, they had lower CGPAs than would be predicted by their academic performance in high school. These findings reveal three characteristics of UofT Engineering: its selectivity and rigor, transnational character of its student population, and complex student diversity. The study also suggests UofT Engineering look into its grading practices.
\end{abstract}

Keywords: engineering pipeline, high schooluniversity pathway, immigration status, academic outcomes

\section{INTRODUCTION}

Students' interests in science, technology, engineering and mathematics (STEM) fields are cultivated as early as elementary and high school [1, 2]. Engineering programs often have "leaky pipelines" in which many qualified students do not eventually enter engineering programs or occupations [3-5]. To attract more students to their field, many engineering schools in Canadian universities have outreach offices that introduce high school students to the engineering field and profession (see examples in [6]) [7].

This paper contributes to the study of student pipelines into engineering programs in two ways. First, we analyzed an integrated data set that longitudinally tracked multiple cohorts of graduates from the Toronto District School Board (TDSB), Canada's largest school board, to the University of
Toronto (UofT), Canada's largest university and the most popular postsecondary destination for TDSB graduates. The TDSB-UofT "pipeline" is likely the largest of its kind in Canada. We used these data to answer the following questions:

- Do UofT engineering students' academic and demographic backgrounds differ from those TDSB graduates admitted to other STEM programs, and all other fields at UofT?

- Do UofT engineering students graduate at different rates and have different GPAs than other students, and if so, what factors are associated with those outcomes?

Our second contribution is to discuss a key finding that tends to be downplayed in the literature on "leaky pipelines": that is, immigrant students are significantly over-represented in some engineering schools. We interpret that finding to reflect the increasingly transnational student population enrolled in Engineering as a field of study, at least in contexts like the UofT and the region of Toronto.

\section{LITERATURE REVIEW}

The metaphor of "leaky pipeline" has been used to describe flows of students in and out STEM fields of studies in postsecondary education, emphasizing in particular the underrepresentation of certain groups, usually those defined by gender and race [5]. Since an early US National Academy of Sciences report [8] introduced the pipeline model, a plethora of literature on "leaky pipelines" has emerged, focusing on two key transitions: that from elementary and secondary education into postsecondary education (PSE); and that from postsecondary education into workplaces. Our literature review focuses on the former transition.

The US-based literature shows that women and some minority students tend to "leak out" of STEM pipelines. Some studies suggest that women students had almost the same level of aspirations for STEM courses as men [9] and had taken as many as, in some cases more, math and science courses as compared to men [10]. However, women were less likely to pursue 
STEM programs as their first choice in PSE and were less decisive about pursuing STEM-related careers [9]. Similarly, a disproportionately lower proportion of African American and Hispanic students earn engineering credentials [11]. Yet, unlike gender differences, those ethnic inequalities can emerge in the US during high school, with Hispanic and African American students having less access to qualified teachers and challenging courses in math and science [11].

Four factors can explain why students choose to pursue a STEM career: psychological, environmental, sociological, and educational [12]. Psychological factors include STEM-related academic abilities, intrinsic motivation and values students attach to STEM-related tasks; environmental factors include role models, peers and class dynamics; sociological factors include peer influence, parental expectations, and prior educational experiences in another educational system with more rigorous STEM curricula; and educational factors include specialized curricula and schools.

In comparison to American studies, Canadian studies reveal both similarities and differences in the situation north of the border. In terms of gender, underpresentation of women in STEM fields in Canada has been persistent for decades despite the steady increase in enrolment by women [13]. Data from 2011 showed that while $61 \%$ of adults aged 25 to 64 with a nonSTEM university degree were women only $33 \%$ of STEM degree holders were women; and women accounted for an increased share of STEM degree holders among younger workers [14]. Moreover, young female immigrants were more likely to hold STEM degrees than were Canadian-born degree holders, suggesting that immigrant status influences the STEM pipeline [14]. Course streaming in Canada occurs in later high school grades, when students can opt out of science and math courses [15]. Thus, Canadian STEM pipelines are likely to better reflect high school students' choices and family supports than deeper structural inequalities. Further, in Canada, high school students' preferences for STEM are highly related to their math abilities, among both men and women, while there were no significant gender difference in science scores [14]. Nonetheless, young women with higher levels of mathematical ability (as measured by PISA) were less likely to pursue STEM

\footnotetext{
${ }^{1}$ We excluded i) students who entered UofT as graduate students; ii) the 2000 and 2001 cohorts due to their high rates of missing data on demographics; and iii) the three youngest cohorts (Grade 9 students) in the falls of 2008,2009 and 2010, as many of those students would not have managed to graduate from UofT by fall 2018.

CEEA-ACEG20; Paper 33
}

Concordia and McGill Universities; June 18-21, $2020 \quad-2$ of $10-$ fields than were young men with lower levels of mathematical ability [14].

Few Canadian studies have examined the impact of immigrants on engineering pipelines, despite the fact that over half $(54 \%)$ of the university-educated STEM graduates in Canada are immigrants, with immigrants representing only $22 \%$ of the Canadian population in 2016 [16]. Our study helped fill this gap by examining differences by gender and immigration status in student pipelines from high school into postsecondary engineering.

\section{METHODS}

Data Source. Data came from a partnership between the TDSB and UofT. Data from several TDSB and UofT sources were merged, including official student records and a survey of student demographics. Those data captured all students who were in Grade 9 in a TDSB school between 2000 and 2010 and later entered UofT between the falls of 2004 and 2018 (see [17] for details). This design captured all students in the TDSB-UofT pathway across 11 cohorts, creating a total sample of 32,302 students. Of those students, 2,483 (7.7\%) were in Engineering ( $0.9 \%$ had missing data for field of study). We then created an analytic sample of 14,286 students from six TDSB Grade 9 cohorts (2002-2007) after excluding three categories of cases, ${ }^{1}$ of which 1,012 (7.1\%) entered UofT Engineering.

Statistical Models. We examined two student outcomes-graduation status and cumulative grade point average (CGPA) - and the following two groups of independent variables:

(1) High School Academics:

- Number of days absent in Grade 9

- Whether or not ever suspended in high school

- Average grades in the last four years of high school

- Grade 9 English, science and math standardized test ${ }^{2}$ scores

- Whether or not passed Grade 10 literacy test on the first attempt

(2) Student Demographics:

- Gender

- $\quad$ Age in Grade 9

- Immigration status: whether born in Canada

\footnotetext{
2 The Education Quality and Accountability Office administers standardized tests to Ontario students in Grades 3,6 and 9, as well as a literacy test in Grade 10.
} 
- Whether or not the student speaks English only at home

- Whether or not a parent went to university

- Whether or not a parent has a professional occupation

- Median income of neighbourhood

- Whether or not the student comes from a two-parent family

Our analysis began with a series of t-tests and Mann-Whitney tests to compare characteristics of engineering students with those in all other UofT fields, and those in other STEM programs. We ran logistic regression and ordinary least squares (OLS) regression models to examine the effects of predictors on the two outcomes.

Data Strengths and Limitations. Our data had several strengths for addressing our research questions. In terms of scope, they covered an entire yet distinctive population, rather than a sample, thus having good generalizability to that population. For the purposes of causal inference, our focus on a single high school-university pathway controlled for a range of unmeasured variables that might confound data that pooled students across an array of school boards and universities, such as variables related to entering universities with different levels of prestige or those that have or do not have an engineering program. Conversely, any findings from this large and urban high school-university pathway may not be generalizable to other kinds of pathways, such as those between smaller and/or rural boards and universities.

\section{FINDINGS}

We next report findings for our two research questions.

\subsection{Bivariate Differences between Engineering Students and Other Students}

Table 1 reports bivariate tests of mean differences for a series of comparisons between Engineering, other STEM students, and students in all other UofT fields. Engineering students had significantly higher graduation rates than other students, with $87 \%$ of engineering students graduating by June 2018, compared to $78 \%$ of other STEM students and $70 \%$ of all other students. Engineering students also had statistically significantly higher CGPAs than did other students, scoring 0.19 points higher than all others ( $21 \%$ of a standard deviation, Cohen's $d=.23$ ) and 0.12 points higher than other STEM students $(14 \%$ of a standard deviation, Cohen's $d=.14$ ). Thus at the bivariate level, engineering students had better university outcomes than others.

In terms of our predictors, Table 1 also shows that engineering students had better high school academic track records than other students, and somewhat different demographics. Compared to all others, engineering students were significantly less likely to ever have been suspended during high school, had fewer days of absence, had far higher average grades ( $64 \%$ of a standard deviation, Cohen's d $=.74$ ), higher Math, English and science test scores, and were more likely to have passed Ontario's Grade 10 literacy on their first attempt.

In terms of demographics, engineering students were far less likely to be female, more likely to selfidentify as Asian, less likely to self-identify as White or Black, and more likely to be immigrants to Canada. Indeed, almost $60 \%$ of engineering students in the TDSB-UofT pathway were immigrants. Engineering students were also significantly more likely to speak a language in addition to English at home, and more likely to come from two-parent households, have a parent who attended university and a parent employed as a professional. There were no statistically significant differences between engineering students and all other students in terms of the median incomes of their neighborhoods when they were in Grade 9.

Many of the same differences emerged in comparisons of engineering students and all other STEM students, with the following exceptions: there were no significant differences in their high school suspension rates, English test scores, and propensities to pass Ontario's Grade 10 Literacy test, self-identify as Asian, speak a language other than English, or come from a two-parent household. Nonetheless, engineering students were more likely than other STEM students to be male, have high average high school grades and math and science test scores, be immigrants to Canada, and have university-educated and professional parents. Thus on average, engineering students had stronger high school academic records than other UofT students, and tended to differ on some socio-demographics, particularly gender and immigration status. 
Table 1: T and Mann-Whitney Tests Comparing Engineering Students to All other UofT Students and to Other STEM Students

\begin{tabular}{|c|c|c|c|c|c|c|c|}
\hline \multirow[t]{3}{*}{ Variable groupings } & \multirow[t]{3}{*}{ Variables } & \multirow{2}{*}{\multicolumn{2}{|c|}{$\frac{\text { Reference group }}{\text { Engineering }}$}} & \multicolumn{4}{|c|}{ Comparison groups } \\
\hline & & & & \multicolumn{2}{|c|}{ All Other Fields } & \multicolumn{2}{|c|}{$\begin{array}{c}\begin{array}{c}\text { All Other STEM } \\
\text { Fields }\end{array} \\
\end{array}$} \\
\hline & & $\%$ & $\begin{array}{l}\text { Mean } \\
(\mathrm{SD})\end{array}$ & $\%$ & $\mathrm{M}(\mathrm{SD})$ & $\%$ & $\mathrm{M}(\mathrm{SD})$ \\
\hline \multirow[t]{2}{*}{ Outcomes } & Graduation status & $87.15 \%$ & & $69.93 \% * * *$ & & $77.52 \% * * *$ & \\
\hline & $\begin{array}{l}\text { Cumulative grade } \\
\text { point average } \\
\text { (CGPA) }\end{array}$ & & $\begin{array}{l}2.84 \\
(.77)\end{array}$ & & $\begin{array}{l}2.65 * * * \\
(.90)\end{array}$ & & $\begin{array}{l}2.72 * * * \\
(.85)\end{array}$ \\
\hline \multirow{7}{*}{$\begin{array}{l}\text { Academic indicators } \\
\text { in high school }\end{array}$} & Suspend & $7.01 \%$ & & $8.9 \% *$ & & $6.37 \%$ & \\
\hline & Absence & & $\begin{array}{l}1.32 \\
(.66) \\
\end{array}$ & & $\begin{array}{l}1.57 * * * \\
(.87) \\
\end{array}$ & & $\begin{array}{l}1.39 * * \\
(.75) \\
\end{array}$ \\
\hline & $\begin{array}{l}\text { Average grades in last } \\
\text { four years }\end{array}$ & & $\begin{array}{l}84.38 \\
(6.56)\end{array}$ & & $\begin{array}{l}78.32 * * * \\
(9.47)\end{array}$ & & $\begin{array}{l}81.75 * * * \\
(7.89)\end{array}$ \\
\hline & English scores & & $\begin{array}{l}3.21 \\
(1.10)\end{array}$ & & $\begin{array}{l}3.07 * * * \\
(1.11)\end{array}$ & & $\begin{array}{l}3.17 \\
(1.10)\end{array}$ \\
\hline & Math scores & & $\begin{array}{l}3.74 \\
(.62) \\
\end{array}$ & & $\begin{array}{l}3.04 * * * \\
(1.13)\end{array}$ & & $\begin{array}{l}3.48 * * * \\
(.86)\end{array}$ \\
\hline & Science scores & & $\begin{array}{l}3.61 \\
(.78)\end{array}$ & & $\begin{array}{l}3.16 * * * \\
(1.05)\end{array}$ & & $\begin{array}{l}3.51 * * * \\
(.84)\end{array}$ \\
\hline & Literacy test & & $\begin{array}{l}1.04 \\
(.19) \\
\end{array}$ & & $\begin{array}{l}1.06^{* * *} \\
(.24) \\
\end{array}$ & & $\begin{array}{l}1.04 \\
(.21) \\
\end{array}$ \\
\hline \multirow[t]{12}{*}{ Demographics } & Age in Grade 9 & & $\begin{array}{l}14.00 \\
(.21) \\
\end{array}$ & & $\begin{array}{l}14.01 \\
(.18) \\
\end{array}$ & & $\begin{array}{l}14.01 \\
(.19) \\
\end{array}$ \\
\hline & Gender - Female & $20.85 \%$ & & $59.92 \% * * *$ & & $53.50 \% * * *$ & \\
\hline & Ethnicity - Asian & $70.71 \%$ & & $57.28 \% * * *$ & & $70.25 \%$ & \\
\hline & Ethnicity - White & $21.99 \%$ & & $26.96 \% * *$ & & $18.50 \% *$ & \\
\hline & Ethnicity - Black & $0.93 \%$ & & $5.55 \% * * *$ & & $3.12 \% * * *$ & \\
\hline & $\begin{array}{l}\text { Ethnicity - Middle } \\
\text { East }\end{array}$ & $3.36 \%$ & & $4.47 \%$ & & $4.73 \%$ & \\
\hline & Immigrant & $59.29 \%$ & & $43.05 \% * * *$ & & $52.82 \% * * *$ & \\
\hline & Speak English only & $21.91 \%$ & & $34.94 \% * * *$ & & $23.16 \%$ & \\
\hline & $\begin{array}{l}\text { Neighbourhood } \\
\text { socioeconomic status }\end{array}$ & & $\begin{array}{l}7.23 \\
(4.71)\end{array}$ & & $\begin{array}{l}7.47 \\
(4.73)\end{array}$ & & $\begin{array}{l}7.24 \\
(4.55)\end{array}$ \\
\hline & Two-parent family & $87.31 \%$ & & $82.58 \% * *$ & & $85.86 \%$ & \\
\hline & $\begin{array}{l}\text { Parent received } \\
\text { university education }\end{array}$ & $68.34 \%$ & & $57.37 \% * * *$ & & $60.80 \% * * *$ & \\
\hline & Professional parent & $45.53 \%$ & & $35.60 \% * * *$ & & $37.84 \% * * *$ & \\
\hline
\end{tabular}

Note: t-tests were performed on continuous variables (those under the columns marked with "Mean (SD)"); Mann-Whitney tests were performed on dichotomous variables (those under the columns marked with "\%").

$* \mathrm{p}<.05 ; * * \mathrm{p}<.01 ; * * * \mathrm{p}<.001$

\subsection{Multivariate Regression Models}

Our multivariate models examined whether these academic and socio-demographic variables predicted entry into UofT Engineering, and whether engineering students had better graduation rates and CGPAs than other students after controlling for the predictors. We used logistic regression to model entry into Engineering and graduation status, since they are binary outcome, and OLS regression to model CGPA, which is continuous. Note that "entry" here reflects both admission decisions and student choice to apply and accept an offer from UofT Engineering.

Table 2 shows results from a logistic regression model that predicted entry into Engineering. The first column shows that overall high school academic performance and particularly Math scores had strong positive effects, controlling for other academic variables; however, English scores had negative effects. Unsurprisingly, students who were stronger in 
Math choose to pursue Engineering while those who were stronger in English chose other fields. These findings are consistent with a recent Statistics Canada report that found that males, on average, performed better in high school math, worse in reading, and the same in science, compared to females; and that math abilities were associated with the choice of STEM fields [14]. Low absenteeism and having been suspended were also positively associated with entry into Engineering. When demographic variables were added (Column 2), females were significantly less likely to enter Engineering controlling for high school academics and other demographics, while immigrants and non-English speakers were more likely. The addition of these variables into the model shrank the effect of being suspended to non-significance, while the coefficients for high school grades and math and English scores remained significant. Adding socioeconomic variables in Column 3 left all of the significant demographic and academic effects intact; having professional parents was positively associated with entry into Engineering. Thus, Table 2 suggests that pursuit of Engineering was predicted by (mostly) strong academic records in high school and by gender, immigration status, and having parents with professional occupations. Results from regressions predicting entry into Engineering vis a vis all other STEM students (not shown) revealed an almost identical pattern.

Table 3 presents coefficients from a logistic regression on status of graduation from UofT. The first column examines the impact of being in Engineering on the odds of graduation before controls were added to the model. It shows that studying in an engineering program almost tripled the odds of graduation compared to all non-engineering students. Adding academic controls in Column 2 shrank those odds almost by half, but engineering students remained significantly more likely to graduate. Adding demographic controls in Column 3 further shrank the Engineering coefficient, though it again remained highly significant. Finally, adding socioeconomic variables in Column 4 had no impact on the Engineering coefficient. Thus, Table 3 suggests that engineering students were significantly likelier than all other students to graduate from UofT controlling for a broad range of demographic, academic and socioeconomic measures. Also noteworthy is that women and students from two-parent families were more likely to graduate from UofT while, curiously, students who speak English only were less likely to graduate than others.

Another set of analyses (not shown) that compared propensities to graduate between engineering students and other STEM students revealed a similar pattern. Without any controls, engineering students' odds of graduating were almost double those of other STEM students. Adding academic controls shrank the odds ratio to 1.6 , though it remained highly significant. Adding demographic controls raised that ratio to 1.67, while adding socioeconomic variables did not impact it. Thus, our analyses showed that engineering students were significantly more likely to graduate than other STEM students controlling for a range of prior academic, demographic and socioeconomic measures.

Table 4 examines the impact of Engineering status on CGPAs. The first column reveals that engineering students on average obtained 0.19 higher points compared to all other students. Adding high school academics in Column 2 had a striking impact: it reversed the direction of the Engineering coefficient indicating that engineering students' CGPAs were, on average, 0.13 lower than others' controlling for high school track records. Adding other demographics in Column 3 further increased that coefficient in a negative direction, while adding socioeconomic variables had no impact. Thus, these models suggest that engineering students, on average, received about .17 points less on their CGPAs than would be predicted by their high school track records and sociodemographics, with an effect size of $-0.15 .^{3}$ Across UofT as a whole, Asian and Black students also received significantly lower CGPAs. An additional analysis that compared engineering students to other STEM students (not shown) revealed a similar pattern of results, though at lesser magnitudes: engineering students attained .09 fewer points on their CGPAs controlling for prior academics and sociodemographics, an effect size of -0.12 .

Table 2: Results from logistic regression analysis on entry into engineering programs

\begin{tabular}{|l|c|c|c|c|c|c|}
\hline \multirow{2}{*}{ Variables } & \multicolumn{2}{c|}{ Column 1 } & \multicolumn{2}{c|}{ Column 2 } & \multicolumn{2}{c|}{ Column 3 } \\
\cline { 2 - 7 } & Odds ratio & SE & Odds ratio & SE & Odds ratio & SE \\
\hline High school cohorts & 1.02 & .02 & 1.04 & .03 & 1.03 & 0.34 \\
\hline Suspend & $1.62^{* *}$ & .23 & 1.05 & .16 & 1.06 & 0.20 \\
\hline Absence & $.90^{*}$ & .05 & 1.00 & .06 & 1.03 & 0.76 \\
\hline
\end{tabular}

\footnotetext{
${ }^{3}$ We calculated effect size by dividing the Engineering coefficient by the standard deviation of CGPA.

CEEA-ACEG20; Paper 33

Concordia and McGill Universities; June 18 - 21, $2020 \quad-5$ of $10-$
} 


\begin{tabular}{|c|c|c|c|c|c|c|}
\hline $\begin{array}{l}\text { Average grades in last four years of } \\
\text { high school }\end{array}$ & $1.09^{* * *}$ & .01 & $1.11^{* * *}$ & .01 & $1.11^{* * *}$ & 0.12 \\
\hline English scores & $.77 * * *$ & .03 & $.84 * * *$ & .04 & $.83 * *$ & 0.05 \\
\hline Math scores & $1.80^{* * *}$ & .12 & $1.48^{* * *}$ & .11 & $1.58^{* * *}$ & .14 \\
\hline Science scores & .95 & .05 & .91 & .06 & .91 & 0.07 \\
\hline Literacy test & 1.02 & .20 & 1.08 & .25 & 1.08 & 0.32 \\
\hline Age in Grade 9 & & & $.61^{*}$ & .13 & $.57^{*}$ & 0.14 \\
\hline Gender - Female & & & $.14 * * *$ & .01 & $.15 * * *$ & 0.02 \\
\hline Ethnicity - Asian & & & 1.15 & .26 & 1.01 & 0.25 \\
\hline Ethnicity - White & & & 1.13 & .26 & .98 & 0.24 \\
\hline Ethnicity - Black & & & .57 & .24 & .52 & 0.27 \\
\hline Ethnicity - Middle East & & & .78 & .24 & .61 & 0.22 \\
\hline Immigrant & & & $1.44 * * *$ & .13 & $1.35^{*}$ & 0.15 \\
\hline Speak English only & & & $.76^{*}$ & .09 & $.69^{* *}$ & 0.09 \\
\hline Neighbourhood SES & & & & & .99 & 0.01 \\
\hline Two-parent family & & & & & 1.34 & 0.20 \\
\hline Parent received university education & & & & & 1.16 & 0.13 \\
\hline Professional parent & & & & & $1.26^{*}$ & 0.12 \\
\hline Constant & $2.36 \mathrm{e}-26$ & $1.14 \mathrm{e}-24$ & $5.91 \mathrm{e}-39$ & $3.26 \mathrm{e}-37$ & $1.14 \mathrm{e}-27$ & $7.50 \mathrm{e}-26$ \\
\hline $\mathrm{N}$ & 13,386 & & 11,416 & & 8,515 & \\
\hline LR chi2(8) & $651.57 * * *$ & & $1138.43 * * *$ & & $868.01 * * *$ & \\
\hline Log likelihood & -3131.13 & & -2415.75 & & -1741.09 & \\
\hline Pseudo R2 & 0.09 & & 0.19 & & 0.20 & \\
\hline
\end{tabular}

${ }^{*} \mathrm{p}<.05 ; * * \mathrm{p}<.01 ; * * * \mathrm{p}<.001$

Table 3: Results from logistic regression analysis on graduation status

\begin{tabular}{|c|c|c|c|c|c|c|c|c|}
\hline \multirow[t]{2}{*}{ Variables } & \multicolumn{2}{|c|}{ Column 1} & \multicolumn{2}{|c|}{ Column 2} & \multicolumn{2}{|c|}{ Column 3} & \multicolumn{2}{|c|}{ Column 4} \\
\hline & $\begin{array}{l}\text { Odds } \\
\text { ratio }\end{array}$ & SE & Odds ratio & SE & Odds ratio & SE & Odds ratio & $\mathrm{SE}$ \\
\hline Engineering & 2.92 & .28 & $1.64 * * *$ & .20 & $1.52 * * *$ & .17 & $1.56 * *$ & 0.21 \\
\hline $\begin{array}{l}\text { High school } \\
\text { cohorts }\end{array}$ & & & $.89 * * *$ & .01 & $.89^{* * *}$ & .01 & $.89 * * *$ & 0.02 \\
\hline Suspend & & & .90 & .07 & 1.00 & .08 & .97 & 0.10 \\
\hline Absence & & & $.87 * * *$ & .02 & $.90 * * *$ & .03 & $.92 *$ & 0.03 \\
\hline $\begin{array}{l}\text { Average grades in } \\
\text { last four years of } \\
\text { high school }\end{array}$ & & & $1.11 * * *$ & .00 & $1.12 * * *$ & .00 & $1.11 * * *$ & 0.01 \\
\hline English scores & & & $.90 * * *$ & .02 & $.90 * * *$ & .02 & $.91 * *$ & 0.03 \\
\hline Math scores & & & .97 & .02 & .95 & .03 & .96 & 0.03 \\
\hline Science scores & & & $.94 *$ & .03 & .95 & .03 & .95 & 0.03 \\
\hline Literacy test & & & .89 & .08 & .88 & .09 & $.77 *$ & 0.10 \\
\hline Age in Grade 9 & & & & & $.74 *$ & .10 & $.79 *$ & 0.13 \\
\hline Gender - Female & & & & & $1.12^{*}$ & .05 & $1.14^{*}$ & 0.06 \\
\hline Ethnicity - Asian & & & & & 1.01 & .11 & .90 & 0.11 \\
\hline Ethnicity - White & & & & & 1.00 & .10 & .87 & 0.10 \\
\hline Ethnicity - Black & & & & & $.75^{*}$ & .10 & .79 & 0.12 \\
\hline $\begin{array}{l}\text { Ethnicity - } \\
\text { Middle East }\end{array}$ & & & & & 1.14 & .17 & .98 & 0.17 \\
\hline Immigrant & & & & & .96 & .05 & .94 & 0.06 \\
\hline $\begin{array}{l}\text { Speak English } \\
\text { only }\end{array}$ & & & & & $.76^{*}$ & .05 & $.72 * * *$ & 0.05 \\
\hline $\begin{array}{l}\text { Neighbourhood } \\
\text { SES }\end{array}$ & & & & & & & 1.01 & 0.01 \\
\hline
\end{tabular}




\begin{tabular}{|c|c|c|c|c|c|c|c|c|}
\hline $\begin{array}{l}\text { Two-parent } \\
\text { family }\end{array}$ & & & & & & & $1.27 * *$ & 0.09 \\
\hline $\begin{array}{l}\text { Parent received } \\
\text { university } \\
\text { education }\end{array}$ & & & & & & & 1.03 & 0.06 \\
\hline $\begin{array}{l}\text { Professional } \\
\text { parent }\end{array}$ & & & & & & & 1.00 & 0.06 \\
\hline Constant & 2.33 & .04 & $2.98 \mathrm{e}+98^{* * *}$ & $8.79 \mathrm{e}+99$ & $1.4 \mathrm{e}+102 * * *$ & $4.5 \mathrm{e}+103^{* * *}$ & $4.8 \mathrm{e}+101 * * *$ & $1.8 \mathrm{e}+103$ \\
\hline $\mathrm{N}$ & 14,166 & & 13,386 & & 11,416 & & 8515 & \\
\hline LR chi2(8) & $156.94 * *$ & & $2179.49 * * *$ & & $1845.85 * * *$ & & $1343.48 * * *$ & \\
\hline Log likelihood & -8430.80 & & -6943.68 & & -5823.94 & & -4337.85 & \\
\hline Pseudo R2 & 0.01 & & 0.1357 & & 0.1368 & & 0.1341 & \\
\hline
\end{tabular}

$* \mathrm{p}<.05 ; * * \mathrm{p}<.01 ; * * * \mathrm{p}<.001$

Table 4: Results from OLS regression analysis on CGPAs

\begin{tabular}{|c|c|c|c|c|c|c|c|c|}
\hline \multirow{2}{*}{ Variables } & \multicolumn{2}{|c|}{ Column 1} & \multicolumn{2}{|c|}{ Column 2} & \multicolumn{2}{|c|}{ Column 3} & \multicolumn{2}{|c|}{ Column 4} \\
\hline & Coefficient & SE & Coefficient & $\mathrm{SE}$ & Coefficient & $\mathrm{SE}$ & Coefficient & $\mathrm{SE}$ \\
\hline Engineering & $.19 * * *$ & .03 & $-.13 * * *$ & .03 & $-.14 * * *$ & .03 & $-.17 * * *$ & .03 \\
\hline High school cohorts & & & -.00 & .00 & .00 & .01 & .00 & .01 \\
\hline Suspend & & & .05 & .03 & .05 & .03 & .06 & .03 \\
\hline Absence & & & $.07 * * *$ & .01 & $.04 * * *$ & .01 & $.04 * *$ & .01 \\
\hline $\begin{array}{l}\text { Average grades in last four } \\
\text { years of high school }\end{array}$ & & & $.06 * * *$ & .00 & $.06^{* * *}$ & .00 & $.06 * * *$ & .00 \\
\hline English scores & & & .01 & .01 & -.02 & .01 & -.02 & .01 \\
\hline Math scores & & & $-.03 * * *$ & .01 & $-.03 * * *$ & .01 & $-.04 * * *$ & .01 \\
\hline Science scores & & & .00 & .01 & .01 & .01 & .01 & .01 \\
\hline Literacy test & & & .00 & .03 & .04 & .03 & .02 & .04 \\
\hline Age in Grade 9 & & & & & $-.10 *$ & .04 & -.08 & .05 \\
\hline Gender - Female & & & & & $-.03 *$ & .02 & -.02 & .02 \\
\hline Ethnicity - Asian & & & & & $-.08 *$ & .03 & $-.11 * *$ & .04 \\
\hline Ethnicity - White & & & & & .05 & .03 & -.00 & .04 \\
\hline Ethnicity - Black & & & & & $-.15 * *$ & .05 & $-.14 * *$ & .05 \\
\hline Ethnicity - Middle East & & & & & .07 & .05 & .06 & .05 \\
\hline Immigrant & & & & & -.03 & .02 & -.04 & .02 \\
\hline Speak English only & & & & & $.06^{*}$ & .02 & $.02 * *$ & .02 \\
\hline Neighbourhood SES & & & & & & & .01 & .00 \\
\hline Two-parent family & & & & & & & .03 & .02 \\
\hline $\begin{array}{l}\text { Parent received university } \\
\text { education }\end{array}$ & & & & & & & .03 & .02 \\
\hline Professional parent & & & & & & & $.04 *$ & .02 \\
\hline Constant & $2.65 * * *$ & .01 & 1.99 & 9.34 & -7.85 & 10.13 & -6.04 & 11.63 \\
\hline $\mathrm{N}$ & 13,355 & & 12,597 & & 10,592 & & 8,029 & \\
\hline F value & $41.03 * * *$ & & $608.87 * * *$ & & $272.55 * * *$ & & $183.83 * * *$ & \\
\hline Adj R-squared & 0.00 & & 0.3028 & & 0.3158 & & 0.3235 & \\
\hline
\end{tabular}

$* \mathrm{p}<.05 ; * * \mathrm{p}<.01 ; * * * \mathrm{p}<.001$

\section{DISCUSSION}

In this section, we discuss five main findings in light of recent literature and the social context of Toronto. First, consistent with virtually all other studies [14-16], females were starkly underrepresented in the TDSB-UofT Engineering pathway, controlling for prior academics and socio- demographics. Since only $21 \%$ of the TDSB-UofT pathway into Engineering (mostly in 2004-2010) was comprised of females, there was clearly a "leaky pipeline" of female students in that pathway. Further data analysis could explore whether females with better academic performance were less likely to pursue Engineering, as has been found at the national level [14]. Nonetheless, our data showed that female

CEEA-ACEG20; Paper 33

Concordia and McGill Universities; June 18 - 21, $2020 \quad-7$ of $10-$ 
engineering students, among UofT female students, were more likely to graduate than males, thus demonstrating the persistence of females on their chosen fields of study, including engineering. But this leak needs to be seen in a broader context. Currently, $35 \%$ of UofT engineering students are female [18]. This is much higher than the cohort average of $21 \%$ among TDSB graduates reported in Table 1, as the enrolment of women in the Faculty has risen in recent years:

Second, rather than ethnicity, immigration status was significantly associated with the likelihood of entry into Engineering, thus confirming the preponderance of immigrant students in high schooluniversity pathway in the field of engineering. This aligns with a pattern in a broader context, wherein immigrants are over-represented in STEM graduates across Canada [16]. In our data, the percentage of immigrant students in Engineering (59\%) was much higher than the $22 \%$ of the population in Canada who reported having ever been landed immigrants in the 2016 census [19]. Our data also showed that overall UofT attracted a higher proportion of immigrant students (43\% in non-engineering programs). Note that students in our pathway are not international students; they instead went to high school in TDSB and thus are mostly Canadian citizens or landed immigrants. We found that students from immigrant families were significantly more likely to enter Engineering, controlling for high school academic records and other demographics. We speculate that immigrants and international students are drawn to STEM fields in general and engineering in particular because of the high valuation placed on those fields in other continents. Leaders of developing nations tend to prioritize engineering as a field over social science and humanities fields as a strategy for building infrastructure and modernizing their economies [20]. Emerging economies, including BRIC countries (i.e., Brazil, Russia, India and China), have increased the proportion of engineering students in postsecondary education more rapidly than have developed countries [21-23]. Among their populaces, engineering is perceived to be a desirable and high status profession [21], while social science and humanities fields have a far lower social profile, with fewer such offerings in universities in developing nations [24]. These factors seem to play a role in flowing more immigrants and international students into Canada's engineering pipeline, particularly in cities like Toronto.

Third, consistent with other literature [13, 15], superior STEM abilities in high school, particularly in math, was a strong predictor of flowing through TDSB's pipeline into UofT Engineering. Our analysis showed that those TDSB students who were admitted to UofT Engineering had better math performance in high school than those who entered other STEM fields; students who scored higher in math in high school were nearly $60 \%$ more likely to pursue engineering programs when controlling for other academic records and demographics. Since Engineering is a competitive field, students need to accumulate a significant amount of educational prowess to enter engineering programs. Both schooling and family background can play important roles in enabling high school students to gain such superior STEM abilities.

Fourth, UofT engineering students had better academic outcomes than other UofT students: when controlling for high school academic performance and other demographics, engineering students were over $50 \%$ more likely to graduate and had better academic performance, as indicated by their CGPAs. These findings demonstrate that UofT engineering programs produce high-performing graduates.

Fifth, UofT engineering students' CGPAs were significantly lower than would be predicted by their previous academic performance, a finding that suggests that the rigour of UofT engineering programs appears to deflate student grades. Nevertheless, engineering students were more likely to graduate than were others. More investigation is needed to explore the factors that contribute to this finding.

Overall, we interpret our findings as highlighting three distinctive characteristics of UofT engineering programs. First, they are highly selective and rigorous, admitting high school students with superior academic records, graduating students at high rates while awarding relatively lower grades. This characteristic means that the engineering pipeline is a narrow one.

Second, students in UofT Engineering are highly transnational. Our data showed that engineering programs attracted a higher proportion of immigrant students than other programs. Moreover, these programs have also attracted students from across the globe: while $27.5 \%$ of its engineering undergraduates in 2018-19 were international students, $40 \%$ of undergraduates lived outside North America before their enrolment at UofT Engineering [18]. This transnational nature can be understood in the contexts of UofT and the city of Toronto. UofT is characteristic of the relatively flat hierarchy of Canadian universities that incentivizes most high school graduates to attend a local university rather than to re-locate to other regions or go abroad for undergraduate studies [25]; the university has thus become one of the major recipients of local students from the Toronto region. On the other hand, UofT also has a substantial proportion of its students from across the world because of its international reputation for research and graduate studies; thus it is a distinctively local-global university [26]. Further, the transnational character also mirrors the city of Toronto where UofT is located. 
Toronto is Canada's largest city, and, for long, a gateway for selective immigration and offers bountiful educational opportunities, with four universities. These social contexts have shaped the transnational character of UofT Engineering's student population. This characteristic suggests that the engineering pipeline appears to be particularly attractive to students with certain cultural backgrounds, such as Asian students, who have overflown the pipeline, so to speak.

Third, a complex context for diversity, equity and inclusion exists within UofT Engineering. Our data show a strong association of immigration status with students' pursuit of Engineering, with an intersection between immigration status and race. Asian students are well over-represented, while both White and Black students are under-represented. In other words, the traditional equivalence between "minority" and "being under-represented" no longer applies within the Faculty, nor across Canadian Universities as a whole, where $40 \%$ of university undergraduates are visible minorities ${ }^{4}$ in contrast to $22 \%$ of Canada's general population [27]. These emerging patterns require that equity and inclusion practices need to adapt if they are to accommodate the needs of all sorts of under-represented students.

These three characteristics of UofT Engineering its selectivity and rigor, the transnational character of its student population, and its complex student diversity - limits generalizability to engineering schools at other Canadian universities. Further investigations of how engineering schools differ from each other can verify whether these three characteristics reflect the broader state of engineering education in Canada.

\section{References}

[1] Brenda M. Capobianco, Brian F. French, and Heidi A. Diefes-Dux, "Engineering identity development among pre-adolescent learners," Journal of Engineering Education, vol. 101, no. 4, pp. 698-716, 2012.

[2] Adam V. Maltese and Christina S. Cooper, "STEM pathways: Do men and women differ in why they enter and exit?," AERA Open, vol. 3, no. 3, pp. 1-16, 2017.

[3] Heather Metcalf, "Stuck in the pipeline: A critical review of STEM literature," InterActions: UCLA Journal of Education and Information Studies, vol. 6, no. 2, 2010.

[4] Alice L. Pawley and Jordana Hoegh, "Exploding pipelines: Mythological metaphors structuring
Our findings have practical implications for parents, high-school teachers and advisors, and for university admissions and outreach offices. All actors need to continue to identify high school students' potential for pursuing engineering studies, and help those qualified make informed decisions. Sharing the characteristics of engineering programs and academic outcomes of engineering students with high school students will help them make informed decisions about their postsecondary education. Outreach offices need to continue their efforts to recruit female students from local high schools, but should also recognize existing patterns of under- and over-representation of various students in Engineering and react accordingly. They should also add social class to their list of demographic markers. Further, UofT Engineering needs to review its grading practices to look into the reasons for engineering student receiving lower CGPAs than predicted based on their academic performance in high school.

Finally, we end by recognizing that further research is needed to verify whether the characteristics of UofT Engineering identified in this study are shared in other engineering schools across Canada. Are the CGPAs of engineering students at other Canadian universities also lower than those expected based on their high school grades? Do student bodies in other Canadian engineering schools also have the demographic patterns we uncovered at UofT Engineering? Answers to these questions will help produce better understandings of high schooluniversity pathways into engineering in Canada.

[5] Gabrielle H. Lyon, Jameela Jafri, and Kathleen St. Louis, "Beyond the pipeline: STEM pathways for youth development," Afterschool Matters, vol. 16, pp. 48-57, 2012.

[6] Riadh W. Y. Habash and Christine Suurtamm, "Engaging high school and engineering students: A multifaceted outreach program based on a mechatronics platform," IEEE Transactions on Education, vol. 53, no. 1, pp. 136-143, 2010.

[7] Linda Katehi, Greg Pearson, and Michael Feder, "Engineering in K-12 education: Understanding the status and improving the prospects," Washington, DC: The National Academies Press, 2009 ,
Available:

\footnotetext{
${ }^{4}$ Visible minority is defined as "persons, other than Aboriginal peoples, who are non-Caucasian in race or non-white in colour" [19]. 
https://www.nap.edu/catalog/12635/engineeringin-k-12-education-understanding-the-status-andimproving.

[8] National Research Council, Engineering infrastructure diagramming and modeling. Washington, DC: National Academies Press, 1986.

[9] Roli Varma and Hahn Hahn, "Gender and the pipeline metaphor in computing," European Journal of Engineering Education, vol. 33, no. 1, pp. 3-11, 2008.

[10] Paul Schreuders, Susan Mannon, and Brian Rutherford, "Pipeline or personal preference: Women in engineering," European Journal of Engineering Education, vol. 34, no. 1, pp.97-112, 2009.

[11] Change the Equation, "Engineering emergency: African Americans and Hispanics lack pathways to engineering," in "Vital Signs: Reports on the Condition of STEM Learning in the U.S," Washington DC: Change the Equation, 2014, Available from https://www.ecs.org/wpcontent/uploads/CTE VitalSigns Engineering V $\underline{\text { 5.pdf. }}$

[12] Rena F. Subotnik, Robert H. Tai, Rochelle Rickoff, and John Almarode, "Specialized public high schools of science, mathematics, and technology and the STEM pipeline: What do we know now and what will we know in 5 years?," Roeper Review, vol. 32, no. 1, pp. 7-16, 2010.

[13] Lesley Andres and Maria Adamuti-Trache, "You've come a long way, Baby? Persistent gender inequality in university enrolment and completion in Canada, 1979-2004," Canadian Public Policy vol. 33, no. 1, pp. 93-116, 2007.

[14] Sarah Jane Ferguson, "Women and education: Qualifications, skills and technology.," in "Women in Canada: A Gender-Based Statistical Report," Ottawa, ON: Statistics Canada, 2016.

[15] Maria Adamuti-Trache and Lesley Andres, "Embarking on and persisting in scientific fields of study: Cultural capital, gender, and curriculum along the science pipeline," International Journal of Science Education, vol. 30, no. 12, pp. 15571584, 2008.

[16] Garnett Picot and Feng Hou, "Skill utilization and earnings of STEM-educated immigrants in Canada: Differences by degree level and field of study," Ottawa, ON: Statistics Canada, 2019, Available from https://www150.statcan.gc.ca/n1/pub/11f0019m/1 1f0019m2019023-eng.htm.

[17] Robert Brown, Scott Davies, and Neil Chakraborty, "The University of Toronto-Toronto District School Board cohort analysis report 1: Introductory findings," Toronto, ON: Data, Equity, and Policy in Education Lab, Ontario Institute for Studies in Education, 2019, Available from https://www .oise.utoronto.ca/depelab/workingpapers/.
[18] Faculty of Applied Science \& Engineering, "Annual report," Toronto, ON: Faculty of Applied Science \& Engineering, University of Toronto, 2019, Available from https://www.engineering.utoronto.ca/files/2019/0 8/UTENG Annual Report 2019 Web.pdf.

[19] Statistics Canada, "Immigration and ethnocultural diversity: Key results from the 2016 Census," Ottawa, ON: Statistics Canada, 2017. Available from https://www150.statcan.gc.ca/n1/dailyquotidien/171025/dq171025b-eng.htm

[20] Russel C. Jones. "Engineering capacity building in developing countries, " Virginia: World Expertise, 2007. Available from http://www.worldexpertise.com/Engineering Cap acity Building in Developing Countries.htm

[21] Cebr for the Royal Academy of Engineering, "Engineering and economic growth: A global view," London, UK: Royal Academic of Engineering, 2016, Available: https://www .raeng.org.uk/publications/reports/en gineering-and-economic-growth-a-global-view.

[22] Martin Carnoy et al., University expansion in a changing global economy: Triumph of the BRICs? . Redwood City, CA: Stanford University Press, 2013.

[23] United Nations Educational Scientific and Cultural Organization, "Engineering: Issues, challenges and opportunities for development," Paris, France: United Nations Educational, Scientific and Cultural Organization, 2010. Available from https://unesdoc.unesco.org/ark:/48223/pf0000189 753.

[24] Maria Charles, "What gender is science?," Contexts, vol. 10, no. 2, pp. 22-28, 2011.

[25] Scott Davies and Floyd Hammack, "Channelling competition in higher education: Comparing Canada and the US," Journal of Higher Education, vol. 76, no. 1, pp. 89-106, 2005.

[26] Glen A. Jones, "Can provincial universities be global institutions? Rethinking the institution as the unit of analysis in the study of globalization and higher education," Higher Education Policy, vol. 21, no. 4, pp. 457-468, 2008.

[27] Universities Canada, "Recent data on equity, diversity and inclusion at Canadian universities," Universities Canada, 2019, Available from https://www.univcan.ca/wpcontent/uploads/2020/02/UC 2019 EDIStats EN.pdf. 